* Corresponding author Phone (+421)907/107866 E-mail address: robert.bucko@ivmk.sk (Ing. Robert Bucko)

Article information Article history: AMS-Volume16-No.1-00147-12 Received 14 January 2012 Accepted 15 March 2012

\section{Epistemic logic and the process of verification used in ambient intelligence home environment}

\author{
Robert Bucko \\ Slovak University of Technology, Bratislava, Slovakia
}

\section{BIOGRAPHICAL NOTES}

Ing. Robert Bucko works at the Slovak University of Technology Bratislava, Faculty of Electrical Engineering and Information Technology.

\section{KEY WORDS}

Epistemic logic, verification, smart home, ambient intelligence

\section{ABSTRACT}

In this work I present the way of using an epistemic logic and the process of knowledge verification in order to control and monitor the object in the given environment. The achievement of correct recognition of situations depends on the system knowledge and how the system responds on the given issue. The aim is to describe and induce an example of using the logical or epistemic procedures.

\section{Introduction}

The world population age is always increasing. It is going to be one of the greatest challenges for the researchers and companies of the 21 st century. The main problem of aging population is that people are not able to take care of themselves even in very simple and daily tasks. First mentioned smart house was in the early 1980s when the intelligent building concept was used. In the concept, the intelligent implementation of consumer electronic devices, electrical equipment, and security devices aiming for the automation of domestic tasks, easy communication, and human friendly control, as well as safety, was proposed. In the earlier development, the idea was oriented to build a smart house environment for ordinary non-disabled persons with the simple purpose of enhancing home comfort. [1] We can find many various solutions using classical mathematical logic in order to monitor or control the subject (person) and responds its needs in given environment. Epistemic logic is based on the knowledge and gained experience. I give a short review of epistemic logic and its possibility of use in ambient intelligence systems.

In case of ambient intelligence systems and verification of process it is necessary to obtain new knowledge during the running processes. Running process means to control and monitor the subject (occupant) in the environment. Al systems could analyze and recognize the conditions using logical procedures and avoid to involuntary situations in monitoring environment. Logical procedures and approaches can use the collected data from sensors, monitoring systems and suggest the solution or indicate any problem.

\section{Epistemic logic definition}

Swedish-Finnish philosopher G.H. von Wright was the first person who wrote about 
epistemic logic in his book An Essay in Modal Logic [2].

Epistemic modifiers are hard to diversify from other kinds of modifiers. Expressions like "can" "impossible" or "cannot" are often used to formulate and define statements about our knowledge and understanding and not the statement what is possible independently of the knowledge [2].

\section{S1: It is possible that the human in my environment needed to call an Emergency}

The statement S1 is not saying that human need is objectively possible, but more or less the need of human is not excluded. If we knew the human needed to call the Emergency we would not say the statement S1. We would say:

\section{S2: Human in my environment needed to call an Emer- gency for sure}

or

\section{S3: I am absolutely sure human in my environment need- ed to call an Emergency.}

Even thought we would distinguish when to say an objectively possible statement and when we say a statement within the meaning of our knowledge. It is difficult to consider what kind of operators is adequate for the statements analysis, especially if it is not given the context. There is a lot of common analogies between epistemic and alethic operators. How nearly the epistemic sentential operators behave the same as (or like) the alethic or modal?

\section{Logic language}

As any other logic the epistemic logic has its own language and semantics as well. Let define the basic principle:

\section{Definition:}

Epistemic logic knows new operators which are not allowed in modal logic. These operators are $K$ (know), $B$ (believe). It can be considered as modal logic extension.

Let $P$ be a non-empty set of propositional variables and $n \in N$ be given. The language $L^{\prime}$ is the smallest superset of $P$ such as:

$\varphi, \psi \in L^{\prime} \Rightarrow \neg \varphi,(\varphi \wedge \psi), K_{i \varphi}, E_{\varphi}, C_{\varphi}, D_{\varphi} \in L^{\prime}(i \leq n)$

These expressions we read as follows: $\mathrm{E} \varphi$ "everyone knows $\varphi$ ", $C \varphi$ "it is common knowledge that $\varphi$ ", $D \varphi$ means " $\varphi$ is distributed knowledge". Epistemic logic gives new options for the system and the system can work on other alternatives and analyze these options deeply depending on the system performance.

$K \varphi A$ - subject (agent) $\varphi$ knows that $A$ is true.

We can define an operator $\mathrm{P}$ by this operator as follows:

$$
P_{\varphi} A \Longleftrightarrow \neg K_{\varphi} \neg A
$$

We read this as $\varphi$ does not know $\neg \mathrm{A}$, or what $\varphi$ knows is not excluded what is possible in $A$.

\section{Example of use}

Three intelligent children are coming back home from the playground. Some of them have mud on their forehead. Mother wants to have a serious talk with the muddy children. None of them knows whether it is muddy or not, but they can all see the others and perceive if they are muddy. It is supposed to all children are perfect logical reasoners. Given issue we know as the muddy children problem. We can see how the epistemic logic can be use to solve this issue.

Their mother said: At least one of you is muddy. If you are muddy, please come forward.

- Three children (agents)

- Operators $(K a, K \beta, K \gamma, B a, B \beta, B \gamma)$

- Available relations $(R a, R \beta, R \gamma)$

- Facts:

\section{- $a$ a is muddy \\ - $b \beta$ is muddy \\ - $c y$ is muddy}

- Children do not know whether they are muddy

- All agents know $a \vee b \vee c$ (mother's statement)

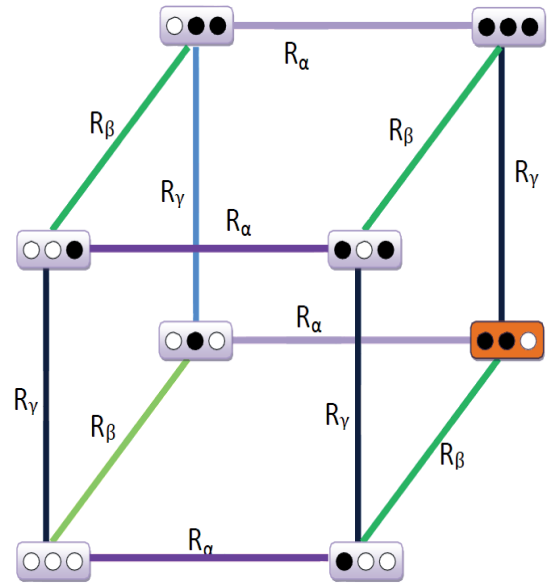

Fig. 1: All the possible statements and transfers among them. Fulfilled circles representing muddy child. Real world is representing by orange color. 
Let say $s=(1,1,0)$ is representing the real world. It means that number 1 represents muddy child forehead and number 0 (zero) the clean one.

1. $M, s \vDash \neg\left(K_{1} m_{1} \vee K_{1} \neg m_{1}\right)$ (child 1 does not know whether it is muddy)

2. $M, s \vDash K_{1} m_{2} \vee K_{1} \neg m_{3}$ (child 1 knows that 2 is muddy and that 3 is not muddy)

3. $M, s \vDash K_{1}\left(m_{2} \wedge \neg K_{2} m_{2}\right) \vee K 1\left(\neg m_{3} \wedge \neg K_{3} \neg m_{3}\right)$ (child 1 knows that 2 is muddy without knowing it and also that 3 is mudless without knowing that)

4. $M, s \vDash K_{1}\left(m_{1} \rightarrow\left(K_{2} m 1 \wedge K_{2} K_{3} m_{1}\right)\right)$ (child 1 knows that, if he is muddy, 2 knows it, and that 2 then also knows that 3 knows it.

\section{Definition of Smart Home}

Home will become a place with the health control center, control system of the most important and usual activities a human can do e. g. energy control, communication center, fun and entertainment programs. The nowadays situations is not prepared to offer these kinds of service, but housing industry prepares to include new technologies into the projects.

Computer companies' sales are about $\$ 30$ billion per year, whereas home construction and renovation market is approaching $\$ 450$ billion per year. Technology companies with the new technologies and research can enter the market and bring better approach, progress and enhancements. [3]

If we look at the smart home system, we have to define some important integration. Smart Home is the term commonly used to define a place or house which uses various controlling and automated systems. There are many various technologies how to monitor and control the system, I would like to focus on the method how to work and analyze the final data which were obtained from the system. It means how to process and make a correct decision from the obtained data. First we need to look at the given environment, what is present and what is expected from the system. Every single home changes in time period and therefore we need to reflect this change and let the system learn the new changed environment. It will be extremely expensive to have prepared developer to control the surrounding environment and react to any modification or variation, so it is better to let the system control it by itself. If we look at the system which has to control, monitor and analyze every single change and modification, it would be reasonable to divide the system into the agents with the pre-compliance responsibility and functionality. We will consider the control system as multi-agent system used mentioned logic.

\section{Using MAS}

Multi-agent system seems to be great to simulate and analyze behavior of the individual systems. Each agent obtains new knowledge and feeds the knowledge database by observing the interaction of other agents (as explained on the above example) and it is associated with a set of other agents. Communication among the agents has to be permanent in order to keep the information flow.

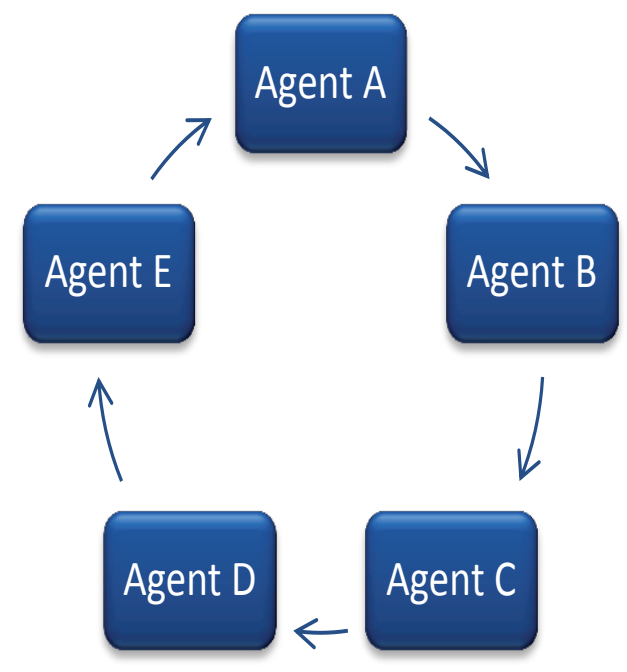

Fig. 2: Multi-agent model using 5 individual agents. Arrows representing the communication flow.

All the agents would have access to the knowledge database in order to prove obtained knowledge.

\section{Decision problem}

The system has to analyze the obtained data and makes a decision. The problem is with the possible alternatives.

Let $A=\left\{A_{1}, A_{2^{\prime}} A_{3^{\prime}} \ldots A_{n}\right\}$ be a set of $n$ alternatives, where $n \geq 2$.

Let $C=\left\{C_{1}, C_{2}, C_{3^{\prime}} \ldots C_{m}\right\}$ be a set of $m$ criteria, where $m \geq 2$.

The decision matrix is composed of $\mathrm{n}$ alternatives and $m$ criteria.

Let $D=\left[D_{i j}\right]_{n \times m}$ where $D_{i j}$ the value of the alternative $A$ correspondingly to criteria $C$,

where $\mathrm{i}=1, \ldots \mathrm{n}$ and $\mathrm{j}=1, \ldots \mathrm{m}$.

Each agent of specific simulation creates the set: 
$\mathrm{AgP}=\left\{\mathrm{AgP}_{1}, \ldots, \mathrm{AgP}_{k}\right\}$, where $\mathrm{k} \geq 2$ and representing the number of agents. Each $\mathrm{AgP}_{k}$ has defined a set of values for the criteria.

Let $W_{\text {Agpi }}=\left\{W_{c 1}, \ldots, W_{c m}\right\}$ be a set of values for $\mathrm{AgP}_{\mathrm{i}}$, where $\sum_{j=1}^{m} W_{c j}=1, W_{c j} \geq 0$, standing for the definition of a multi-criteria problem.

There are several classifications of solving the problem and decision making models. [4]

The proposed scheme combines the ideas mentioned above and showing the phase of choosing correct alternative.

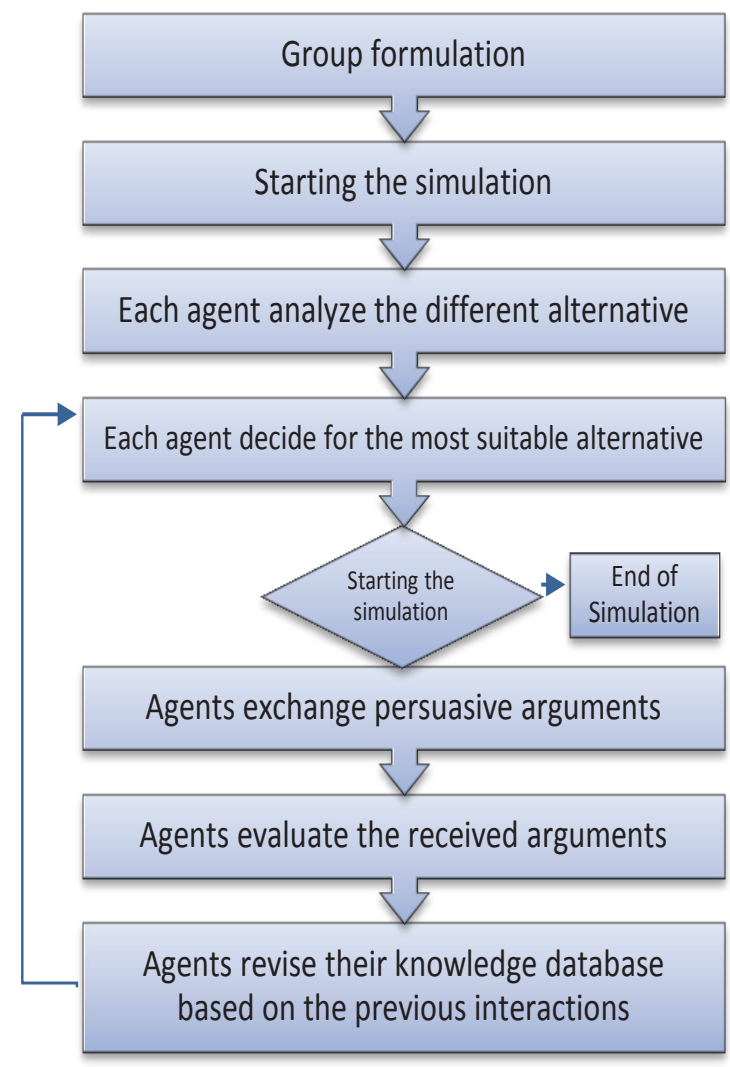

Fig. 3: Decision protocol showing how the agents choose the most preferred alternative.

\section{The argument Exchange}

Another problem can be found if we look at the process of argumentation among the agents. During this process the agents can exchange the following statements:
- Requesting
- Refusing
- Accepting
- Requesting with the argument

All these statements have some specifications which can be done in many various implementations, but I can illustrate a general proposal.

\section{Requesting}

If the agent receives this statement, it is requesting some performance from the other agent/s. It can be provided with the argument if needed. Each agent is trying to give the request to others and provide the best solution based on the obtained knowledge.

\section{Refusing}

The agent is telling to the others that it cannot accept the requirement and it is going to refuse the request. In the figure below you can see the communication between two agents and how they can exchange the arguments.

\section{Accepting}

Acceptance of the request received from the other agent. Acceptance is a statement which means the agents found the solution of the issue in given environment.

In the figure 4 you can see only the communication between two agents. It is necessary note that the real argumentation alternate among many agents including other processes. You can find some arguments which the agents use to persuade the others about the right decision and it is based on the human negotiations. We can find various models of selection the arguments in order to persuade other agent, most of them came from the actual situation of the human world.[5] Here are the six arguments: threats; promise of the future reward and appeals; appeal to past reward; appeal to counter example; appeal to prevailing practice; appeal to self interest. The agent try to generate persuasive arguments used the knowledge database.

\section{Verification process}

When the multi-agent systems reach the goal and final state the process of finding solution is over. Next step is to verify if the solution which has been found is correct and true. The verification of MAS is showing that the system is correct and has no mistakes based on the given conditions and requirements. Currently, the most used and successful approach of verification of any computer system is model checking. Model checking is a set or collection of techniques which automatically analyzing the system and showing the system satisfaction. This method of verification was adopted as a standard procedure for the system quality. 


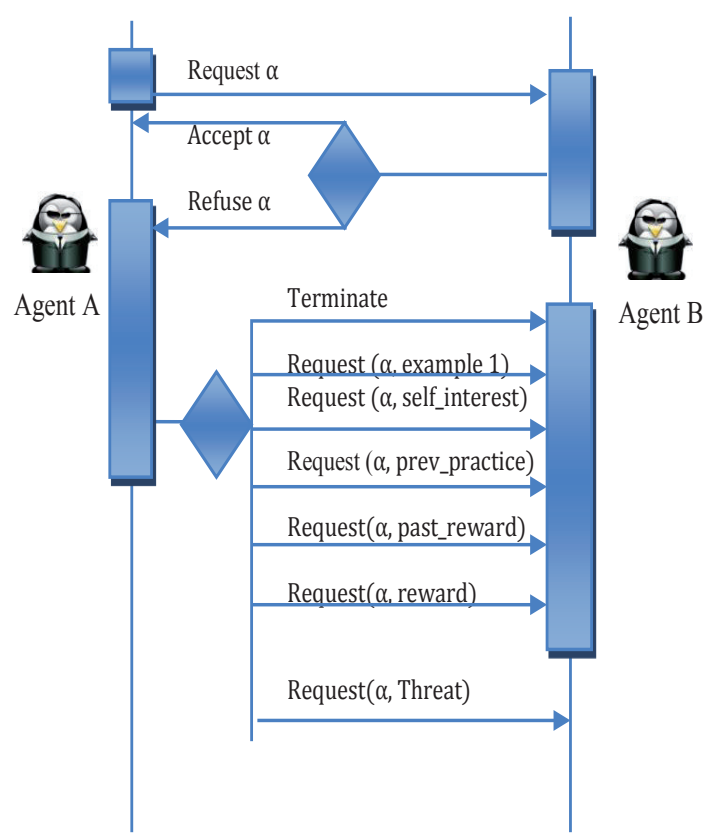

Fig. 4: Communication between two agents. Exchanging the arguments.

\section{Conclusion}

I have shown the basic principle of epistemic logic and explain the specific operators on logical example of muddy children. The work pointing at the decision making problem, showing a decision protocol and describe the statements of exchanging the arguments in communication among the agents in MAS. In final part I outline the concept of model checking as one of the most used technique for verification systems. Many researchers have been focused on the decision problem not just in MAS, but in other decision maker systems as well. Multi-agent systems are more and more used in intelligence applications and hardware realizations. Therefore I think it will be worth continuing to the deeper analyze and measuring the performance of the reaching decisions, verification and satisfaction of the solution.

\section{Acknowledge}

It is a pleasure to thank those who made this thesis possible, my supervisor doc. RNDr. Jaroslav Fogel, PhD..

\section{References}

[1] STEFANOV, D.H.; ZEUNGNAM BIEN;WON-CHUL BANG. 2004. The smart house for older persons and persons with physi- cal disabilities: structure, technology arrangements, and perspectives. [on line]. IEEE Trans. On neural systems and rehabilitation engineering, vol. 12,

[2] WRIGHT, G.H. VON. AN ESSAY IN MODAL LOGIC . s.I. NorthHolland, 1953.

[3] MASSACHUSETTS INSTITUTE OF TECHNOLOGY (MIT BLDG NE18-4FL), House_n Research Consortium. 2005 [on line]: Accessible on: <http://architecture.mit.edu/house_n/documents/HousenConsortiumResearchTopics.pdf>

[4] H. SIMON. The New Science of Management Decision. Harper and Row, New York, 1960.

[5] J. FRENCH, B. RAVEN. The bases of social power. In: Studies in social power. The University of Michigan, pp.150-167, 1959.

[6] G. MARREIROS, C. RAMOS E J. NEVES. Multi-Agent Approach to Group Decision Making through Persuasive Argumentation. 6th International conference on argumentation. Amsterdam, 2006. 